\title{
Cumulative Sum Charts and Its Healthcare Applications; A Systematic Review
}

\author{
R.Sasikumar ${ }^{* 1}$ and S.Bangusha Devi ${ }^{2}$
}

Department of Statistics, Manonmaniam Sundaranar University, Tirunelveli, India. Corresponding Author: sasikumarmsu@gmail.com

Received: September 18, 2013 / Revised: February 28, 2014 / Accepted: March 20, 2014

(C) IAppStat-SL 2013

\begin{abstract}
Application of Statistical process control (SPC) is increasing being advocated in health science as a valuable quality improvement tool. The use of control charts in health science related applications differs somewhat from industrial. In SPC, cumulative sum (CUSUM) charts rather than Shewhart chart are used to detect smaller shifts in the process. In order to improve the detection capability relative to that of the Shewhart control charts, several control charts have been developed such as CUSUM charts, Exponentially weighted moving average(EWMA) charts and Sequential probability ratio test (SPRT) etc. Among them, the CUSUM charts are popular and effective. The CUSUM procedure has recently made its appearance in the field of medicine. The main purpose of this paper is to elucidate the common uses of CUSUM charts in healthcare and to describe different forms of CUSUM charts for monitoring performance over time when the outcome is related to health science.
\end{abstract}

Keywords: Cumulative sum chart, binomial CUSUM, bernoulli CUSUM, risk adjusted CUSUM chart, sequential probability ratio test, healthcare surveillance.

\section{Introduction}

The CUSUM chart was proposed by Page in 1954 for monitoring small shifts in mean in any production process. In the industrial site, CUSUM charts are used to detect small persistent process changes [9]. CUSUM charts rather than the Shewhart chart are usually applied to biological data. CUSUM techniques have been used in the field of medicine for the last 35 years and control charts have been used atleast 50 years. Williams et al., [16] has first proposed the uses of the CUSUM in the field of medicine and CUSUM chart for monitoring surgical performance is documented by De Leval et al. [3]. In the field of medicine, CUSUM procedures in clinical chemistry were used by Nix et al., [10] and to monitor rare congenital malformations by Gallus et al. [4]. CUSUM chart can be updated after each procedure, is applicable to outcomes for individual surgeons and provides a method of real time monitoring of performance. CUSUM chart is a graphical representation of the trend 
in the outcome of a series of consecutive procedures. Acceptable level of performance, the CUSUM curve is flat while the unacceptable levels of performance, the curve slope upward or downward and eventually crosses a decision interval. While this occurs, the CUSUM chart indicates unsatisfactory performance. Average run length (ARL) allows the user to set the sensitivity of the alarm. ARL is the expected number of measurements between false alarms when the processes are still in control. When CUSUM is used to monitoring an industrial process, only one ARL is set, to trigger an inquiry. In the industrial process, samples are taken for testing frequently, often every few seconds, so that the ARL may be set at the level of thousands or even tens of thousands. In the clinical situation, data are examined usually yearly or monthly, and an out of control situation cannot be tolerated for long, so the ARL must be set to a more sensitive level. In the subsequent sections we discussed about design of the CUSUM chart for a normal mean and various CUSUM charts for attribute data that are used for bio and healthcare surveillance.

\section{Design of the CUSUM chart for a normal mean}

A CUSUM chart is a plot of the cumulative differences between successive values and a target value. Consider the sequence $\left\{\mathrm{x}_{\mathrm{i}}\right\}_{i=1}^{N}$ are the observations in a process. When the process is incontrol, $x_{i}$ is distributed according to Normal distribution with mean $\mu_{0}$ and standard deviation $\sigma$. Based on the observed data and target value $\mu_{0}$, the upper and lower CUSUMs are given below. In such a scheme, for the $i^{\text {th }}$ observation $\mathrm{x}_{\mathrm{i}}$, the upper or positive CUSUM $\mathrm{C}^{+}$accumulations deviation above target

$$
\mathrm{C}_{\mathrm{i}}^{+}=\max \left[0, \mathrm{x}_{\mathrm{i}}-\left(\mu_{0}+\mathrm{K}\right)+\mathrm{C}_{\mathrm{i}-1}^{+}\right]
$$

and the lower or negative CUSUM $\mathrm{C}^{-}$accumulations deviations below target.

$$
\mathrm{C}_{\mathrm{i}}^{-}=\max \left[0,\left(\mu_{0}-\mathrm{K}\right)-\mathrm{X}_{\mathrm{i}}+\mathrm{C}_{\mathrm{i}-1}^{-}\right] .
$$

Here $\mathrm{K}$ is the reference value and the value of $\mathrm{K}$ is the halfway distance from the target $\mu_{0}$ to the out of control value of the mean $\mu_{1}$. Based on $\mu_{0}$ and $\mu_{1}$ the shift is defined as

$$
K=\frac{\left|\mu_{1}-\mu_{0}\right|}{2} .
$$

If either $\mathrm{C}_{\mathrm{i}}^{+}$or $\mathrm{C}_{\mathrm{i}}^{-}$exceeds $H$ then the process is considered as out of control. The decision interval is usually taken as $\mathrm{H}=5 \sigma$.

Consider an example for a CUSUM chart for the detection of a shift in mean [9]. The first 20 observations are drawn from a normal distribution with mean $\mu_{0}=10$ and standard deviation $\sigma=1$. The last 10 observations come from a normal distribution with mean $\mu_{1}=11$ and standard deviation $\sigma=1$, so a shift of $1 \sigma$ occurred. The CUSUM control scheme was designed to detect a shift of with reference value 
$k=0.5$ and control limit $\mathrm{H}=5$. From Figure 2.1 it can be seen that the Shewhart control chart is failed to detect small shift from the last 10 observations. Also it can be seen that the CUSUM chart generates an alarm at observation 29. From the CUSUM chart in Figure 2.2, it is observed that the first 20 observations of the CUSUM tend to drift slowly. In this case maintaining values near zero. But in the last 20 observations, the mean has shifted, a strong upward trend developed.

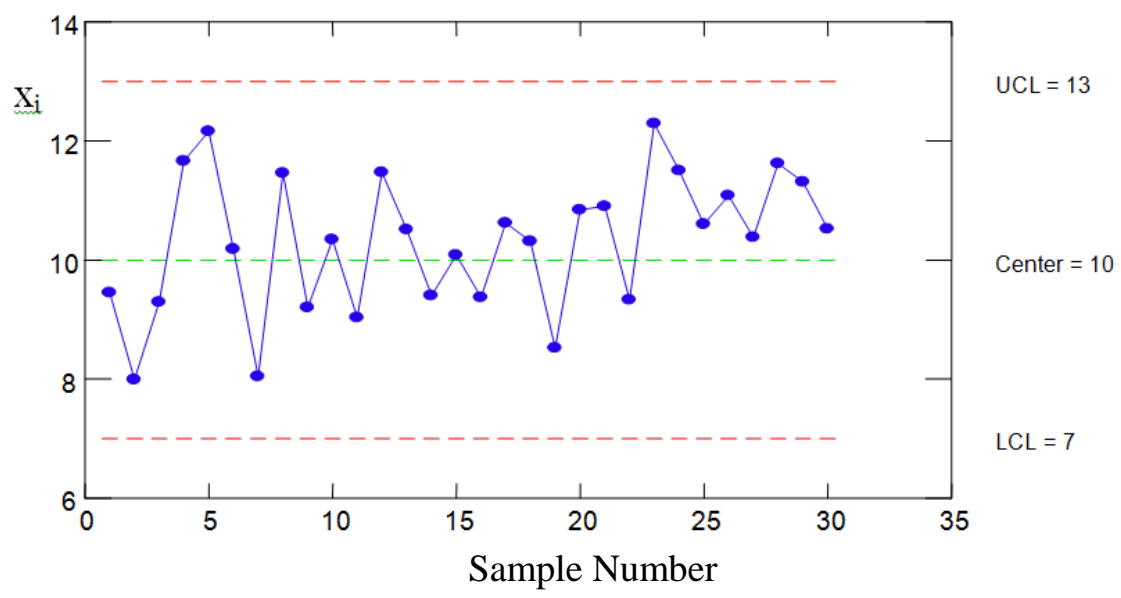

Figure 2.1: Shewhart chart based on data taken from Montgomery (2009)

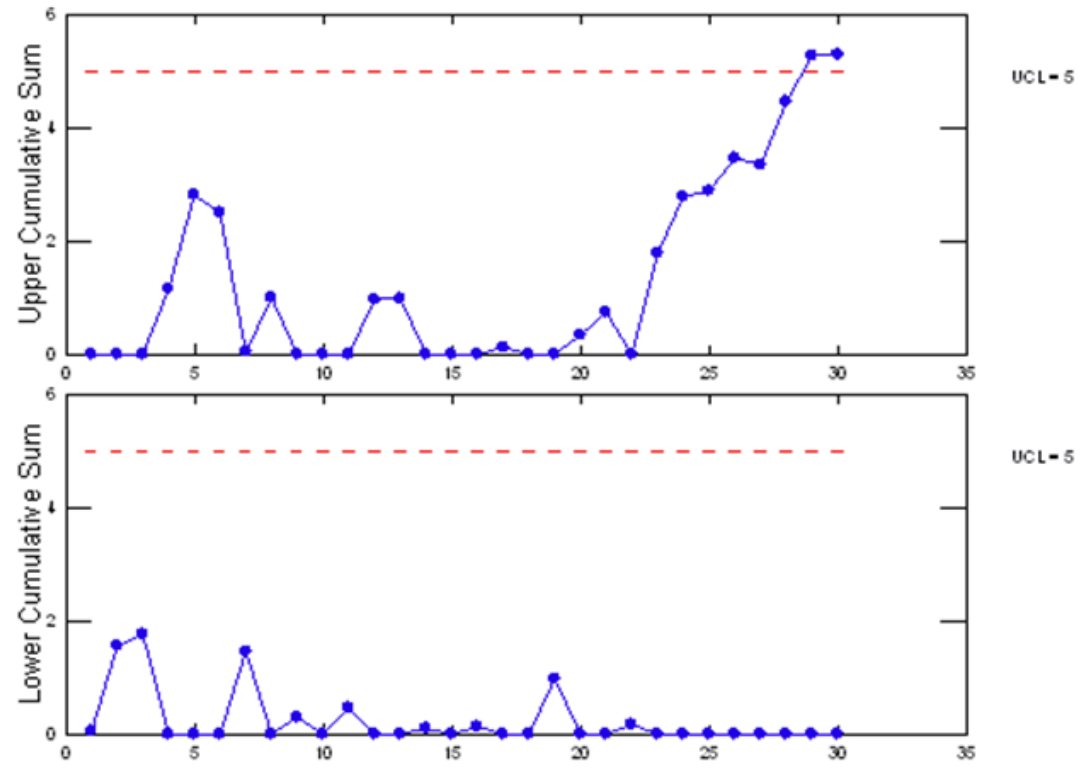

Sample Number

Figure 2.2: CUSUM chart based on data taken from Montgomery (2009) 


\section{Description of various types of CUSUM charts for attribute data}

To analyze the biological data, CUSUM chart play a vital role. Most of the output of the medical data is attribute data. Many charts have been developed which are the extension of CUSUM to analyze the biological data. They are CUSUM chart for binomial data, CUSUM chart for Bernoulli data, Cumulative failure chart, Sequential probability ratio test (SPRT) and etc. Risk adjustment is essential when monitoring healthcare procedures performed on a wide variety of patients. A number of risk adjusted (RA) control chart methods have been proposed $[1,5,12]$. They are the variable life-adjusted display(VLAD)[8] and the equivalent cumulative Risk adjusted mortality(CRAM) chart, the RA resetting sequential probability ratio test (RSPRT)[12] and the RA Bernoulli CUSUM chart[13,14] and etc.

\subsection{Binomial CUSUM}

For numeric data one can apply binomial CUSUM. The outcome of each trial is success (or) failure. The combines result of all these trials is a count number, either the total sum of the trials resulting in success, or the total sum of trials resulting in failure. Given the binomially distributed variable with probability $\mathrm{p}_{0}$ and a defined sample size $(X \sim \operatorname{Bin}(n, p))$, the upper CUSUM $C^{+}$for the upward shift from the incontrol probability $\mathrm{p}_{0}$ to the out-of-control probability $\mathrm{p}_{1}$ of the $\mathrm{i}^{\text {th }}$ observation $x_{i}$ is calculated as

$$
\mathrm{C}_{\mathrm{i}}^{+}=\max \left[0, \mathrm{C}_{\mathrm{i}-1}^{+}+\mathrm{x}_{\mathrm{i}}-\mathrm{K}^{+}\right] .
$$

The lower CUSUM C $\mathrm{C}^{-}$for the upward shift from the in-control probability $\pi_{0}$ to the out-of-control probability $\pi_{1}$ of observation $\mathrm{x}_{\mathrm{i}}$ is calculated as

$$
\mathrm{C}_{1}^{-}=\max \left[0, \mathrm{C}_{\mathrm{i}-1}^{+}-\mathrm{x}_{\mathrm{i}}-\mathrm{K}^{-}\right]
$$

with $\mathrm{C}_{0}^{+}=\mathrm{C}_{0}^{-}=0$. The upward and downward reference values $\mathrm{K}^{+}$and $\mathrm{K}^{-}$ respectively are of the form nk with $\mathrm{k}$ a per unit reference value only depending on $\pi_{0}$ and $\pi_{1}$ and defined by

$$
k=\frac{r_{1}}{r_{2}}=\frac{1}{r}
$$

where $r_{1}=-\log \left(\frac{1-p_{1}}{1-p_{0}}\right)$ is a scaling factor, $r_{2}=\log \left(\frac{p_{1}\left(1-p_{0}\right)}{p_{0}\left(1-p_{1}\right)}\right)$ is a tuning factor and $\mathrm{r}$ is an integer. Similar to the CUSUM for normal mean, the control limit $\mathrm{H}$ can be defined based on a desired in control average run length $\left(\mathrm{ARL}_{0}\right)$ and out of control $\left(\mathrm{ARL}_{1}\right)$. 


\subsection{Bernoulli CUSUM}

If the observations become individually, then we can use CUSUM chart directly on the individual observations, without using any grouping of the observations into samples. The Bernoulli CUSUM chart use individual data to plot appoint on the control chart. The statistic for Bernoulli CUSUM for the upward shift from the incontrol probability $\mathrm{p}_{0}$ to the out-of-control probability $\mathrm{p}_{1}$ of the $\mathrm{i}^{\text {th }}$ observation $\mathrm{x}_{\mathrm{i}}$ is calculated as

$$
\mathrm{C}_{\mathrm{i}}=\left[\max \left(0, \mathrm{C}_{\mathrm{i}-1}\right)+\left(\mathrm{x}_{\mathrm{i}}-\mathrm{K}\right)\right],
$$

with $\mathrm{C}_{\mathrm{i}}=0$ and reference value $\mathrm{k}$ is same as defined in Binomial CUSUM. The control limit $\mathrm{H}$ and ARLs are determined theoretically by simulation, by solving an integral equation approximation or by a Markov chain approximation.

The ARL to signal is obtained by the corrected diffusion and it is

$$
A R L_{0} \approx \frac{\exp \left(h^{*} r_{2}\right)-h^{*} r_{2}-1}{\left|r_{2} p_{0}-r_{1}\right|}
$$

where $\mathrm{h}^{*}$ is an adjusted value of $\mathrm{h}$ and was obtained by the formula

$$
\mathrm{h}^{*}=\mathrm{h}+\varepsilon_{\left(\mathrm{p}_{0}\right)} \sqrt{\mathrm{p}_{0} \mathrm{q}_{0}}
$$

where $q_{0}=1-p_{0}$ and If $0.01 \leq p \leq 0.05$ then

$$
\begin{gathered}
\left.\varepsilon_{(p)} \approx 0.41-0.0842(\log (p))-0.0391(\operatorname{lo}(p))^{3}-0.00367(\log (p))^{4}-0.000008 \log (p)\right)^{7} \\
\varepsilon(p) \approx \frac{1}{3}\left(\sqrt{\frac{1-p}{p}}-\sqrt{\frac{p}{1-p}}\right) .
\end{gathered}
$$

If $0<p<0.01$ then

$$
\varepsilon_{(p)} \approx \frac{1}{3}\left(\sqrt{\frac{1-p}{p}}-\sqrt{\frac{p}{1-p}}\right) .
$$

\subsection{Cumulative mortality plot}

This is a most intuitive form of the CUSUM chart. In the graph of the cumulative mortality chart plots, patients are on the horizontal axis and cumulative number of deaths are on the vertical axis. The observed outcome takes only the values 0 or 1 . To aid the detection of changes in underlying mortality, alert lines are placed on the graph reflecting the false-positive and false-negative rates based on the underlying acceptable mortality rate. In CUSUM charts, boundaries are not constructed by using the standard deviation but by the accepted $\left(\mathrm{p}_{0}\right)$ and the unaccepted $\left(\mathrm{p}_{1}\right)$ failure rate, respectively, according with the type I error rate $(\alpha)$ and the type II error rate $(\beta)$. The following formula presents the calculation for the alert lines 


$$
\begin{aligned}
& a=\left(\frac{1-\beta}{\alpha}\right) \\
& b=\left(\frac{1-\alpha}{\beta}\right) \\
& P=\ln \left(\frac{p_{1}}{p_{0}}\right) \\
& Q=\ln \left(\frac{1-p_{0}}{1-p_{1}}\right) \\
& h_{0}=\frac{a}{P+Q} \\
& h_{1}=\frac{b}{P+Q} \\
& s=\frac{Q}{P+Q}
\end{aligned}
$$

where $h_{0}$ and $h_{1}$ are the lower control limit and the upper control limit respectively. The acceptable line and unacceptable line for cumulative failure chart are $\mathrm{sn}-\mathrm{h}$ and $\mathrm{sn}+\mathrm{h}$ are respectively. The choice of the error rate is free, but one should realize that it has an influence on the false-positive and false-negative conclusions. It is thus possible to use lower error rate values for the construction of an alert line and higher values for the construction of an alarm or caution line. In medicine, both error rates are usually made equal to 0.1 .

\subsection{Risk adjusted CUSUM chart}

The principle of a RA CUSUM is the same as that of the non-RA CUSUM chart described by Steiner et al. [14]. RA CUSUM chart [13] is used to detect an increase or decrease in mortality or both. RA CUSUM chart can be considered as a case of the resetting SPRT chart that is used raptly to monitor a process. Since the difference in RA CUSUM chart is that for a patient with a high mortality risk (40\%), surviving the operation, we are rewarded 0.40 points and thus more than for a low-risk patient. If this high risk patient dies, however, we are only punished by $-(1-0.40)=-0.60$ points, thus less than for a low-risk patient. The benefit of the RA CUSUM chart is that in case of a series of high-risk patients there is no signal of decrement in the performance. In the RA CUSUM chart two charts are used simultaneously, one to detect an increase and one to detect a decrease in mortality. If the chart is designed to detect an increase in mortality, the sum is reset to 0 when it becomes negative. The CUSUM is calculated as

$$
S_{i}=\max \left\{0 ; S_{i-1}+W_{i}\right\} .
$$

If it is designed to detect a decrease in mortality, the sum is reset to 0 when it becomes positive. The CUSUM is calculated as

$$
S_{i}=\min \left\{0 ; S_{i-1}-W_{i}\right\}
$$

where $W_{t}=\left\{\begin{array}{l}\ln \left(\frac{O R_{1}}{1-p_{i}+O R_{1} p_{i}}\right) \text { when the patient survives } \\ \ln \left(\frac{1}{1-p_{i}+O R_{1} p_{i}}\right) \text { when the patient dies }\end{array}\right.$

\subsection{Sequential probability ratio test}

All the charts originate the SPRT that was developed to choose between two hypotheses by sequential testing. SPRT is to use a varied sample size which is 
determined dynamically. It is a sequential test of null hypothesis $H_{0}: p=p_{0}$ against $H_{1}: p=p_{1}$. For each item, $X_{i}=1$ if the $i^{\text {th }}$ item is defective and $X_{i}=0$ otherwise. The SPRT statistic is given by

$$
\mathrm{S}_{\mathrm{i}}=\mathrm{S}_{\mathrm{i}-1}+\mathrm{W}_{\mathrm{i}} \text {. }
$$

The limits of the SPRT chart is given by in terms of two error rates $\alpha$ and $\beta$ (probabilities for type I and II errors). The SPRT requires specifying two constants $a$ and $b, b<a$. The limits are calculated as follows:

$$
a=\log \left(\frac{\beta}{1-\alpha}\right) \quad \text { and } \quad b=\log \left(\frac{1-\beta}{\alpha}\right) .
$$

The following rules are used for sampling and making decisions to accept or reject $H_{0}$. If $\mathrm{b}<\mathrm{S}_{\mathrm{i}}<\mathrm{a}$, then we continue the sampling. If $\mathrm{S}_{\mathrm{i}}=\mathrm{a}$, then we stop the sampling and reject $H_{0}$. If $\mathrm{S}_{\mathrm{i}}=\mathrm{b}$, then we stop the sampling and accept $H_{0}$.

\subsection{Cumulative observed minus expected failure chart}

Cumulative observed minus expected failure graph are intuitive because changes in gradient are more immediately apparent. This chart is more intuitive because it is easier to identify changes in the failure rate. The graph moves upward if it decreases. If boundary lines are draw on a cumulative observed minus expected failure chart, care needs to be taken to specify clearly the hypothesis being tested and to calculated appropriate boundary lines. It is mathematically defined as

$$
C_{t}=C_{t-1}+\left(X_{t}-X_{0}\right),
$$

where $\mathrm{X}_{\mathrm{t}}$ is the outcome measurement for subject $\mathrm{t}$, and $\mathrm{X}_{0}$ is the baseline reference rate.

\subsection{Variable life adjusted display}

The VLAD or CRAM is the chart that displays cumulative difference between expected and observed numbers of mortality versus patient sample. VLAD or CRAM charts are analogous to the cumulative observed minus expected failure chart. Like the cumulative mortality plot, the observed outcome takes a value 0 or 1 . VLAD chart provides easy trend interpretation. It moves upward if the failure rate increases above that predicted by the risk model, moves downward if the rate decreased and oscillates around 0 if performance is consistent with predicted risks (i.e.) acceptable. When the operative mortality rate is consistent with acceptable and expected levels of mortality, the chart will oscillate round the value of zero. A VLAD is a curve that plots cumulative $(\mathrm{E})$ - cumulative $(\mathrm{O})$ events:

$$
V_{n}=\sum_{i=1}^{n} E_{i}-\sum_{i=1}^{n} O_{i} .
$$


Expected outcome $(E)$ can be calculated as:

$$
E=\frac{e^{p}}{1+e^{p}} .
$$

Using the logistic regression to predict a risk coefficient $(p)$ for a patient for a set of $n$ covariates:

$$
\operatorname{logit}(\mathrm{p})=\beta_{0}+\sum_{\mathrm{i}=1}^{\mathrm{n}} \beta_{\mathrm{i}} \mathrm{x}_{\mathrm{i}}
$$

VLAD limits can be calculated from:

For the lower limit the CUSUM of the $\mathrm{n}^{\text {th }}$ observation $\left(C_{n}\right)$ with the corresponding weight $W_{n}$ is given by:

$$
\begin{gathered}
C_{0}=0 \text { and } \\
C_{n}=\max \left\{C_{n-1}+W_{n}, O\right\},
\end{gathered}
$$

where

$$
\mathrm{W}_{\mathrm{n}}=\mathrm{O}_{\mathrm{n}} \log \rho-\log \left(1+(\rho-1) \mathrm{E}_{\mathrm{n}}\right),
$$

and $\rho$ is the ratio of risk under the alternative and null hypotheses.

The lower VLAD limit $\left(L_{n}\right)$ can then be calculated by the expression:

$$
\mathrm{L}_{\mathrm{n}}=\mathrm{V}_{\mathrm{n}}+\left(\mathrm{C}_{\mathrm{n}}-\mathrm{h}\right) / \log \rho
$$

where $\mathrm{h}$ is a control limit signifying when the CUSUM signals.

if $V_{n}$ intersect with $L_{n}$, the limit is reset to $Z_{n}$ by:

$$
\mathrm{Z}_{\mathrm{n}}=\mathrm{L}_{\mathrm{n}}+\mathrm{h} / \log \rho .
$$

For the upper limit, the CUSUM is modified to be:

$$
\mathrm{C}_{\mathrm{n}}=\min \left\{\mathrm{C}_{\mathrm{n}-1}-\mathrm{W}_{\mathrm{n}}, 0\right\},
$$

and the limit is modified to be:

$$
\mathrm{L}_{\mathrm{n}}=\mathrm{V}_{\mathrm{n}}-\left(\mathrm{C}_{\mathrm{n}}+\mathrm{h}\right) / \log \rho
$$

\section{Discussions}

CUSUM charts have different applications in the field of medicine. In health science in different situations CUSUM charts adopted. For example, surgical performance, doctor's performance, rare event in medical context, etc. Steiner et al., [13] introduced RA CUSUM procedure that adjusts for each patient's pre-operative risk of surgical failure through the use of a likelihood-based scoring method. This article provides a logical way to accumulate evidence over many patients, while adjusting for a changing mix of patient characteristics that significantly affect the risk. This method is a generalization of Bernoulli CUSUM chart. 
Rogers et al.,[1] described Cumulative failure chart, SPRT chart, Cumulative observed minus expected failure graph, VLAD or CRAM chart, RA SPRT chart for two types of database that are cardiac surgery database (Surgical failure after offpumb $\mathrm{CABG}(\mathrm{OPCAB})$, cardiothoracic transplantation database (30-day mortality after orthotropic heart transplantation in adults). They used different forms of CUSUM charts that are VLAD and RA SPRT for monitoring performance over time when the outcome of interest is binary. Also, they explained how the charts should be interpreted and recommend ways the charts should be used. Rogers et al., [1] point out that for the RA CUSUM rather than the SPRT. Collins et al., [2] discussed different types of cumulative chart that are cumulative mortality plot, VLAD, cumulative case-mix, funnel plot for the gastro-oesophageal surgery. Because no one surgical tool should ideally be used, instead a combination of methods should be used to monitor surgical outcomes. The cumulative mortality plot is simple but is impractical for rapid detection in changes in the mortality rate. Thabani Sibanda et al., [15] developed Observed minus Expected as well as the two-sided log-likelihood CUSUM chart for monitoring deliveries for nulliparous and multiparous women. Also he detected periods of increased rates of low Apgar scores for each of nulliparous and multiparous cohorts. Lim et al., [7] used CUSUM tool for the monitoring doctor's performance. They demonstrated the utility of upward CUSUM charting in monitoring the quality performance of doctor's from five disciplines in performing a variety of procedures. He also stated that CUSUM is a useful tool for personal audit at an individual and local level.

\section{Conclusion}

The performance of the control charts which are discussed above can be measured and compared by ARL performance. Process monitoring with control charts is an important component within an overall process evaluation and improvement framework in healthcare. This paper highlights the application of CUSUM charts in bio and health surveillance. Further research in a prospective setting is however required in order to fully evaluate these tools. The use of these techniques deserves further attention in the context of prospecting monitoring clinical outcomes.

\section{References}

1. C. A. Rogers, Massimo Caputo, Gianni D. Angelini, Saravana Ganesh J, Robert S. Bonser, Barnaby C. Reeves. (2004). Control chart methods for monitoring cardiac surgical performance and their interpretation. The Journal of Thoracic and Cardiovascular Surgery,128,811-819,

DOI: $10.1016 /$ j.jtcvs.2004.03.011

2. Collins G.S, Jibawi A, McCulloch P. (2010). Control chart methods for monitoring surgical performance: A case study from gastro-oesophageal surgery, Science Direct, 37,473-480. 
3. De leval, Marc R., Francois K, Bull C, Brawn W.B and Spiegelhalter D. (1994). Analysis of a cluter of surgical failures. The Journal of Thoracic and Cardiovascular Surgery, 104, 914-924.

4. Gallus G., Mandelli C., Marchi M and Radaell G. (1986). On surveillance methods for congenitial malformations. Statistics in Medicine, 5, 565-571, DOI: $10.1002 / \operatorname{sim} .4780050603$

5. Grigg O, Farewell V.(2004). An overview of risk adjusted charts. Journal of the Royal Statistical Socity, 167, 523-539,

DOI: 10.1111/j.1467-985X.2004.0apm2.x

6. Grigg OA, Farewell VT, Spiegelhalter DJ. (2003). Use of risk-adjusted CUSUM and RSPRT charts for monitoring in medical contexts. Statistical Methods in Medical Research, 12,147-170.

7. Lim T.O, Soraya A, Ding L.M and Morad Z. (2002). Assessing doctor;s competence: application of CUSUM technique in monitoring doctors' performance. International Journal for Quality in Health Care, 14, 251-258.

8. Lovegrove J, Valencia O, Treasure T, Sherlaw-Jhonson C, Gallivian S. (1997). Monitoring the results of cardiac surgery by variable life display. Lancet, 316,1697-1700.

9. Montgomery D.C. (2009). Introduction to Statistical Quality Control.6 ${ }^{\text {th }}$ edn. New York: Jhon Wiley and Sons.

10. Nix A.B., Rowlands R.J. and Kemp K.M. (1986). Internal quality control in clinical chemistry: a teaching review. Statistics in Medicine , 6, 425 -440.

11. Page E.S (1954). Continuous Inspection Schemes. Biometrika, 41, 100-115.

12. Spiegelhalter D, Grigg O, Kinsman R, Treasure T. (2003). Risk-adjusted sequential probability ratio tests: applications to Bristol, Shipman, and adult cardiac surgery. International Journal for Quality in Health Care, 15,7-13,

DOI: $10.1093 /$ intqhe/15.1.7

13. Steiner SH, Cook RJ, Farewell VT, Treasure T. (2000). Monitoring surgical performance using risk-adjusted cumulative sum charts. Biostatistics, 1, 441452, DOI: $10.1093 /$ biostatistics/1.4.441

14. Steiner SH, Cook RJ, Farewell VT. (2001). Risk-adjusted monitoring of binary surgical outcomes. Medical Decision Making, 21,163-169, DOI: $10.1177 / 02729890122062451$

15. Wald A. (1945). Sequential tests in industrial statistics. Annals of Mathematical Statistics, 350,1128-30.

16. Williams S. M., Parry B.J. and Schlup M.M.(1992). Quality control: an application of the CUSUM. British Medical Journal , 304, 1359-1361. 